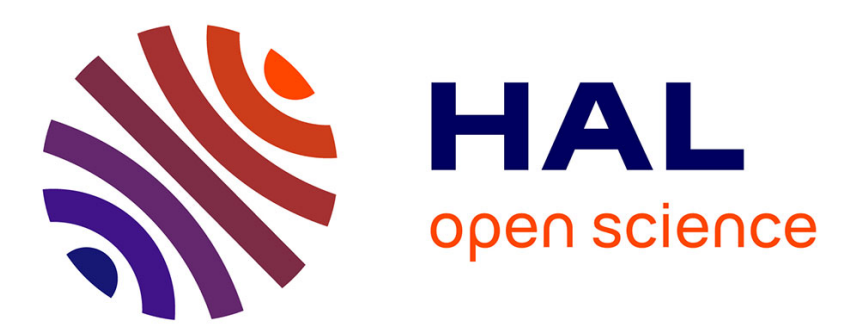

\title{
Some Incipient Techniques For Improving Efficiency in Computational Mechanics
}

\author{
Amine Ammar, Francisco Chinesta
}

\section{To cite this version:}

Amine Ammar, Francisco Chinesta. Some Incipient Techniques For Improving Efficiency in Computational Mechanics. International Journal of Material Forming, 2008, 1, p.1083-1086. 10.1007/s12289008-0207-9 . hal-00742480

\section{HAL Id: hal-00742480 \\ https://hal.science/hal-00742480}

Submitted on 16 Oct 2012

HAL is a multi-disciplinary open access archive for the deposit and dissemination of scientific research documents, whether they are published or not. The documents may come from teaching and research institutions in France or abroad, or from public or private research centers.
L'archive ouverte pluridisciplinaire HAL, est destinée au dépôt et à la diffusion de documents scientifiques de niveau recherche, publiés ou non, émanant des établissements d'enseignement et de recherche français ou étrangers, des laboratoires publics ou privés. 


\title{
Some Incipient Techniques For Improving Efficiency in Computational Mechanics
}

\author{
A. Ammar ${ }^{1}$, F. Chinesta ${ }^{2}$ \\ ${ }^{1}$ Laboratoire de Rhéologie, INPG, UJF, CNRS (UMR 5520) \\ BP 53, Domaine universitaire, 38041 Grenoble Cedex 9
}

e-mail: Amine.Ammar@ujf-grenoble.fr;

${ }^{2}$ LMSP ENSAM Paris

151 Bd de l'Hopital, 75013 Paris

e-mail: Francisco.Chinesta@paris.ensam.fr

\begin{abstract}
This contribution presents a review of different techniques available for alleviating simulation cost in computational mechanics. The first one is based on a separated representation of the unknown fields; the second one uses a model reduction based on the Karhunen-Loève decomposition within an adaptive scheme, and the last one is a mixed technique specially adapted for reducing models involving local singularities. These techniques can be applied in a large variety of models.
\end{abstract}

Key words: Model Reduction, Separated representation, Karhunen-Loève decomposition.

\section{INTRODUCTION}

Models encountered in computational physics and engineering, in particular computational mechanics, usually involve too many degrees of freedom, too many simulation time-steps, too many iterations (non-linear models, optimization or inverse identification), too many particles (in atomistic descriptions) or simply excessive simulation time (for example when simulation in real time is envisaged). Some examples of those scenarios are encountered in computational chemistry involving large time scale differences (reaction versus diffusion), direct numerical simulation of fluid flows (analysis of turbulence), kinetic theory descriptions of solid and fluids (including complex fluids) that usually involve multi-dimensional models with the associated curse of dimensionality problematic, virtual surgery, mechanical systems involving localized behavior, ...

In our former works (see [1] and the references therein) some new strategies of model reduction and computational time saving have been proposed. In what follows the main ideas of those techniques are revisited.

\section{GENERAL FORM OF THE PROBLEM}

Let consider a general field $\Psi$ involving two variables $r$ and $s$ (each one defined on a different space of dimension 1,2 or 3 ). Let consider two operators $\mathcal{K}$ and $\mathcal{L}$ respectively associated to the variables $r$ and $s$.

$$
\mathcal{K}(\Psi(r, s))+\mathcal{L}(\Psi(r, s))=0
$$

For example, if we address the heat equation $\Psi$ represents the temperature, $r$ and $s$ are respectively the time and the physical coordinates, $\mathcal{K}$ is the first order derivative with respect to time, and $\mathcal{L}$ is the Laplace operator. However, Eq. (1) can also represent more complex models. Thus, in steady state kinetic theory models $\Psi$ represents the conformational distribution function, and $r$ and $s$ the physical and the conformational coordinates. In such models the first differential operator is defined in the physical space, accounting for the advective effects, and the second one is related to the induced microstructural evolution that sometimes is modeled from and advection-diffusion differential operator. In other models such as the ones encountered in quantum mechanics, one find a Laplace operator 
defined in the conformation space that in this case is related to the physical space of each particle (electrons or nuclei). Thus, the resulting model results highly multidimensional, making difficult its numerical solution.

In the bead spring chain model (proposed to represent the mechanics of macromolecules) the variables involved are the connector vectors defining the contour length of the chain. The kinetic equation in this case involves, in addition to the general operators of equation (1), other "coupling" operators involving in a coupled way the derivatives with respect to different conformation coordinates.

For the sake of simplicity, from now on we are focusing on the solution of the generic equation (1).

\section{MULTIDIMENSIONNAL APPROACH}

The solution of equation (1) in the multidimensional case, in which a grid (or a mesh) of the domain $\Omega=$ $\Omega_{r} \cup \Omega_{s}$ becomes too expensive or simply forbidden, needs for new advanced strategies. The existing strategies (sparse grids or stochastic techniques) allow addressing moderate multidimensional models. In the highly multidimensional case alternative strategies are needed. One possibility lies in defining separated representations and tensor product approximation basis:

$$
\Psi(r, s)=\sum_{i} \alpha_{i} F_{i}(r) G_{i}(s)
$$

In order to build up this form, a series of projection and enrichment stages are considered.

The projection stage consists to find, for $n$ given terms of the series the best set of the alphacoefficients associated to $F_{1}, G_{1}, \cdots, F_{n}, G_{n}$.

The enrichment stage consists in finding the best functions $R(r), S(s)$ with respect the problem variational formulation.

The computer implementation of these steps needs for a discrete representation of all the functions: $F_{i}(r), G_{i}(s), R(r)$ and $S(s)$ using for example a 1D, 2D or 3D finite element interpolation in their associated domains $\Omega_{r}$ and $\Omega_{s}$. We use for this purpose the vectors $\mathbf{N}$ and $\mathbf{M}$ containing the shape functions associated with each space. Finally $\mathbf{F}_{i}, \mathbf{G}_{i}$, $\mathbf{R}$ and $\mathbf{S}$ represent the nodal description of the associated functions. We define the following matrix related to the variational formulation equation (1):

$$
\begin{array}{ll}
\mathbb{M}=\int_{\Omega_{r}} \mathbf{M M}^{T} d \Omega_{r}, & \mathbb{N}=\int_{\Omega_{s}} \mathbf{N} \mathbf{N}^{T} d \Omega_{s} \\
\mathbb{K}=\int_{\Omega_{r}} \mathbf{M} \mathcal{K} \mathbf{M}^{T} d \Omega_{r}, & \mathbb{L}=\int_{\Omega_{s}} \mathbf{N} \mathcal{L} \mathbf{N}^{T} d \Omega_{s}
\end{array}
$$

These integrals take into account the specific character of each operator. For example integration by parts is used in second order operators, upwinding for stabilizing advective terms, ...

\subsection{Projection stage}

In this stage we consider the test functions given by

$$
\Psi^{*}(r, s)=\sum_{i}^{n} \alpha_{i}^{*} F_{i}(r) G_{i}(s)
$$

which writes in the discrete form:

$$
\Psi^{*}(r, s)=\sum_{i}^{n} \alpha_{i}^{*} \mathbf{F}_{i}^{T} \mathbf{M G}_{i}^{T} \mathbf{N}
$$

Using the discrete form of the $\Psi$ function:

$$
\Psi(r, s)=\sum_{i}^{n} \alpha_{i} \mathbf{M}^{T} \mathbf{F}_{i} \mathbf{N}^{T} \mathbf{G}_{i}
$$

the variational formulation of the equation (1) results

$\sum_{i=1}^{n} \sum_{j=1}^{n} \alpha_{i}^{*} H_{i j} \alpha_{j}=0$

where

$$
H_{i j}=\mathbf{F}_{i}^{T} \mathbb{K} \mathbf{F}_{j} \cdot \mathbf{G}_{i}^{T} \mathbb{N} \mathbf{G}_{j}+\mathbf{F}_{i}^{T} \mathbb{M} \mathbf{F}_{j} \cdot \mathbf{G}_{i}^{T} \mathbb{L} \mathbf{G}_{j}
$$

This system must be solved taking into account some boundary or normality conditions. For example, we could consider a first function product verifying the boundary conditions, and then the other ones must vanish on the domain boundary (this condition is enforced in the enrichment stage). In this case we must enforce $\alpha_{1}=1$.

\subsection{Enrichment stage}

In this stage we consider the unknown field given by

$\Psi(r, s)=\sum_{i} \alpha_{i} F_{i}(r) G_{i}(s)+R(r) S(s)$

being the test functions

$\Psi^{*}(r, s)=R^{*}(r) S(s)+R(r) S^{*}(s)$

That writes in a discrete form as

$$
\Psi^{*}(r, s)=\left[\begin{array}{ll}
\mathbf{R}^{* T} & \mathbf{S}^{{ }^{*} T}
\end{array}\right]\left(\begin{array}{c}
\mathbf{N}\left(\mathbf{S}^{T} \mathbf{M}\right) \\
\left(\mathbf{R}^{T} \mathbf{N}\right) \mathbf{M}
\end{array}\right)
$$

The weak form of the equation (1) becomes, in the framework of a fixed point strategy, in:

- For a given $\mathbf{S}$ (or for a test function in the form of $\left.\Psi^{*}(r, s)=R^{*}(r) S(s)\right)$ 
$\left(\mathbb{K} \mathbf{S}^{T} \mathbb{N} \mathbf{S}+\mathbb{M} \mathbf{S}^{T} \mathbb{L} \mathbf{S}\right) \mathbf{R}=$

$-\sum_{i=1}^{n} \alpha_{i}\left(\mathbb{K} \mathbf{F}_{j} \mathbf{S}^{T} \mathbb{N} \mathbf{G}_{j}+\mathbb{M} \mathbf{F}_{j} \mathbf{S}^{T} \mathbb{L} \mathbf{G}_{j}\right)$

- For a given $\mathbf{R}$ (or for a test function in the form of $\left.\Psi^{*}(r, s)=R(r) S^{*}(s)\right)$

$\left(\mathbf{R}^{T} \mathbb{K} \mathbf{R} \mathbb{N}+\mathbf{R}^{T} \mathbb{M} \mathbf{R} \mathbb{L}\right) \mathbf{S}=$

$-\sum_{i=1}^{n} \alpha_{i}\left(\mathbf{R}^{T} \mathbb{K} \mathbf{F}_{j} \mathbb{N G}_{j}+\mathbf{R}^{T} \mathbb{M} \mathbf{F}_{j} \mathbb{L} \mathbf{G}_{j}\right)$

It must be noticed that the number of the degrees of freedom involved in such non linear solution is the sum of the degrees of freedom involved in each discretization (instead the product resulting in mesh based discretization strategies).

Finally, the new approximation functions $\mathbf{F}_{n+1}$ and $\mathbf{G}_{n+l}$ are obtained from $\mathbf{R}$ and $\mathbf{S}$ after normalization:

$\mathbf{F}_{n+1}=\mathbf{R}\left(\mathbf{R}^{T} \mathbb{M} \mathbf{R}\right)^{-\frac{1}{2}}$

$\mathbf{G}_{n+1}=\mathbf{S}\left(\mathbf{S}^{T} \mathbb{N} \mathbf{S}\right)^{-\frac{1}{2}}$

\section{ADAPTIVE MODEL REDUCTION}

In this section the particular case of $\mathcal{K}=\frac{\partial}{\partial t}$, where $t$ denotes the time, is considered. Let $(t, x)$ be the couple of the two variables involved. In the finite element framework, Eq. (1) is usually solved within an incremental procedure. The unknown field at time $t$ is noted by $\Psi^{t}(x)$ and is associated with the vector representation $\boldsymbol{\Psi}^{t}$ on $\Omega_{x}$. The weak form of the problem (1), using the notation introduced previously, writes

$\boldsymbol{\Psi}^{* T} \mathbb{N} \dot{\boldsymbol{\Psi}}+\boldsymbol{\Psi}^{* T} \mathbb{L} \boldsymbol{\Psi}=\mathbf{0}$

To obtain the evolution of the unknown field during the time an implicit or explicit time integration scheme could be used. But in general, the high number of degrees of freedom involved, makes difficult the problem solution at each time step when semi-implicit or fully-implicit schemes are considered. The main idea of the Karhunen-Loève decomposition is to represent the unknown field using a reduced number of functions expressed on the whole domain $\Omega_{x}$.

\subsection{Karhunen-Loève décomposition}

This decomposition lies in looking for the optimal functions $\phi(x)$ to represent the $P$ discrete fields $\Psi^{n}$ $(n=1, . ., \quad P)$. These functions are obtained by maximizing

$$
\lambda=\frac{\sum_{n=1}^{P}\left[\sum_{i=1}^{N} \phi_{i} \Psi_{i}^{n}\right]^{2}}{\left[\sum_{i=1}^{N} \phi_{i}\right]^{2}}
$$

where $N$ is the total number of nodes in the domain $\Omega_{x}$.

The maximization of $\lambda$ with respect to the functions $\phi$ is equivalent to enforce $\delta \phi=0$, from which it results:

$\sum_{i=1}^{N}\left\{\sum_{j=1}^{N}\left[\sum_{n=1}^{P} \Psi_{i}^{n} \Psi_{j}^{n} \phi_{j}\right] \delta \phi_{i}\right\}=\lambda \sum_{i=1}^{N}\left\{\phi_{i} \delta \phi_{i}\right\}, \quad \forall \delta \phi$

whose matrix form writes

$\delta \phi^{\mathrm{T}} \mathbf{k} \phi=\lambda \delta \phi^{\mathrm{T}} \phi, \forall \delta \phi \Rightarrow \mathbf{k} \phi=\lambda \phi$

where

$$
\mathbf{k}=\mathbf{Q Q}^{\mathbf{T}}, \quad \mathbf{Q}=\left(\begin{array}{cccc}
\Psi_{1}^{1} & \Psi_{1}^{2} & \ldots & \Psi_{1}^{\mathbf{P}} \\
\Psi_{2}^{1} & \Psi_{2}^{1} & \ldots & \Psi_{2}^{\mathbf{P}} \\
\vdots & \vdots & \ddots & \vdots \\
\Psi_{\mathbf{N}}^{1} & \Psi_{\mathbf{N}}^{2} & \ldots & \Psi_{\mathbf{N}}^{\mathbf{P}}
\end{array}\right)
$$

\subsection{A posteriori model reduction}

The time discretization of equation (15) writes:

$\mathbb{N} \boldsymbol{\Psi}^{n+1}=\mathbf{P}^{n}$

that in the case of using an explicit strategy $\mathbf{P}^{n}=(\mathbb{N}-\mathbb{L}) \Psi^{n}$

From the solutions $\boldsymbol{\Psi}^{n}$ one could extract the most significant eigenfunctions (the ones related to the highest eigenvalues): $\phi_{1}, \phi_{2}, \ldots, \phi_{r}$. These functions are stored into a matrix $\mathbf{B}$ which dimensions are $(N, r)$ (in general $r<<N$ ). This matrix allow to describe the evolution of the unknown field using a reduced number of degrees of freedom $(r$ instead $N)$. Thus, writing

$$
\boldsymbol{\Psi}^{n}=\mathbf{B} \mathbf{a}^{n}
$$

equation (19) reduced to:

$\underbrace{\mathbf{B}^{T} \mathbb{N} \mathbf{B}}_{(r, r)} \mathbf{a}^{n+1}=\underbrace{\mathbf{B}^{T} \mathbf{P}^{n}}_{(r, 1)}$

\subsection{A priori model reduction}

The main drawback of the a posteriori model reduction is the necessity to make at least one simulation using the fully finite element description. Besides, one could address the question related to 
the validity of the basis B. The purpose of the "a priori" model reduction [2] is the possibility of defining an adaptive strategy to build up the reduced basis during the model time evolution.

Consequently, we must check the quality of the reduced solution at certain time steps. For this purpose we compute the residual, as proposed in [3]

$$
\mathbf{R}=\mathbb{N} \boldsymbol{\Psi}^{n+1}-\mathbf{P}^{n}=\mathbb{N} \mathbf{B a}^{n+1}-\mathbf{B}^{T} \mathbf{P}^{n}
$$

If the norm of this residual exceeds a given tolerance the basis must be enriched using some functions of the Krylov subspaces generated by the residual, and then the solution evolution is recomputed using the just updated approximation basis. This procedure continues until reaching convergence (that is, a small enough residual norm).

It is obvious that successive enrichments could increase the number of the approximation functions. To avoid this unfavorable effect, a Karhunen-Loève decomposition is performed when the convergence of the enrichment procedure is reached.

\section{TOWARDS A NEW HYBRID STRATEGY}

This last section deals with models involving localized singularities or discontinuities, being the solution far from these singular regions smooth enough. We proved that evolving discontinuities do not accept efficient reduced descriptions. However, one expect that efficient simulations could be achieved by coupling reduced descriptions in the smooth regions and a fully finite element description in the zones involving singularities.

Let $\Omega_{2}$ be the small region of interest where localized singularities exist and $\Omega_{1}$ the remaining part of the domain, verifying

$$
\begin{aligned}
& \Omega_{1} \cup \Omega_{2}=\Omega_{x} \\
& \Omega_{1} \cap \Omega_{2}=\varnothing
\end{aligned}
$$

Each node of the discretization belongs to a single subdomain. The discrete finite element description $\mathbb{N} \dot{\boldsymbol{\Psi}}+\mathbb{L} \boldsymbol{\Psi}=\mathbf{0}$ is now rewritten by introducing a decomposition of the unknown vector: $\boldsymbol{\Psi}_{1}$ and $\boldsymbol{\Psi}_{2}$ which are the respective degrees of freedom in the domains $\Omega_{1}$ and $\Omega_{2}$. Thus the finite element problem could be rewritten as

$$
\left[\begin{array}{ll}
\mathbb{N}_{11} & \mathbb{N}_{12} \\
\mathbb{N}_{21} & \mathbb{N}_{22}
\end{array}\right]\left(\begin{array}{l}
\dot{\boldsymbol{\Psi}}_{1} \\
\dot{\boldsymbol{\Psi}}_{2}
\end{array}\right)+\left[\begin{array}{ll}
\mathbb{L}_{11} & \mathbb{L}_{12} \\
\mathbb{L}_{21} & \mathbb{L}_{22}
\end{array}\right]\left(\begin{array}{l}
\boldsymbol{\Psi}_{1} \\
\boldsymbol{\Psi}_{2}
\end{array}\right)=\mathbf{0}
$$

Over the domain $\Omega_{1}$ the "a priori" model reduction described in the previous section could be applied. A set of reduced basis function are incorporated in the matrix $\mathbf{B}$. A model reduction over $\Omega_{1}$ is made thanks to the next definitions

$$
\begin{aligned}
& \mathfrak{n}_{11}=\mathbf{B}^{T} \mathbb{N}_{11} \mathbf{B} \\
& \mathfrak{l}_{11}=\mathbf{B}^{T} \mathbb{L}_{11} \mathbf{B} \\
& \boldsymbol{\Psi}_{1}=\mathbf{B} \mathbf{a}_{1}
\end{aligned}
$$

which allows writing the initial matrix system (24) as

$$
\begin{aligned}
& \mathfrak{n}_{11} \dot{\mathbf{a}}_{1}+\mathfrak{l}_{11} \mathbf{a}_{1}=-\mathbf{B}^{T} \mathbb{N}_{12} \dot{\boldsymbol{\Psi}}_{2}-\mathbf{B}^{T} \mathbb{L}_{12} \boldsymbol{\Psi}_{2} \\
& \mathbb{N}_{22} \dot{\boldsymbol{\Psi}}_{2}+\mathbb{L}_{22} \boldsymbol{\Psi}_{2}=-\mathbb{N}_{21} \mathbf{B} \dot{\mathbf{a}}_{1}-\mathbb{L}_{21} \mathbf{B} \mathbf{a}_{1}
\end{aligned}
$$

Thus at least in the region $\Omega_{1}$ the computing cost has been significantly reduced. The residual calculation is performed over the whole domain $\Omega_{x}$ and for the enrichment of the basis, only the values of the residual over $\Omega_{1}$ are retained.

\section{REFERENCES}

1. A. Ammar, B. Mokdad, F. Chinesta et R. Keunings. A New Family of Solvers for Some Classes of Multidimensional Partial Differential Equations Encountered in Kinetic Theory Modeling of Complex Fluids. Part II: Transient Simulation Using Space-Time Separated Representation. Journal of Non-Newtonian Fluid Mechanics.

2. Ammar, D. Ryckelynck, F. Chinesta, R. Keunings On the reduction of kinetic theory models related to finitely extensible dumbbells. Journal of Non-Newtonian Fluid Mechanics, 134, 136-147, (2006).

3. D. Ryckelynck. A Priori Hyperreduction Method: an Adaptive Approach. Journal of Computational Physics, 202, 346-366 (2005). 